This paper is published in the open archive of Mid Sweden University

DIVA http://miun.diva-portal.org

with permission of the publisher

Citation for the peer-reviewed published paper:

Norgren S, Höglund H. Irreversible long fibre collapse at high temperature refining in a TMP system : Effects on fibre and surface properties. ; Nordic Pulp \& Paper Research Journal. 2009;24(1):19-24.

URL to article at publishers site:

http://dx.doi.org/10.3183/NPPRJ-2009-24-01-p019-024 


\title{
Irreversible long fibre collapse at high temperature TMP reject refining - Effects on fibre surface properties
}

\author{
Sven Norgren and Hans Höglund, Mid Sweden University, Sundsvall
}

KEYWORDS: Collapse, ESCA, High temperature, Irreversible, Refining, Reject, Roughening, Split fibre

SUMMARY: Effects on fibre and sheet properties of preheating to temperature well above the softening temperature of lignin in refining of spruce TMP reject were evaluated. This study indicates that an improved surface smoothness can be attained on TMP-based wood-containing papers through hightemperature reject refining. Under these conditions the degree of irreversible fibre collapse increased. Results from tests of the fibre-wall, fibre surface characteristics, pulp yield and sheet brightness show only marginal differences at high temperatures compared to standard reject refining. Experiments in which laboratory sheets were moistened showed that a high degree of irreversible fibre collapse correlates with surface smoothness.

\section{ADDRESS OF THE AUTHORS: Sven Norgren}

(sven.norgren@miun.se) and Hans Höglund

(hans.hoglund@miun.se): Mid Sweden University, FSCN,

Fibre Science and Communication Network, SE-851 70

Sundsvall, Sweden.

Corresponding author: Sven Norgren

Favourable surface properties can be attained on softwood TMP-based sheets, when most of the thick-walled long fibres are collapsed (Forseth, Helle 1996, 1998; Forseth et al. 1997; Reme 2000). Under such conditions a large proportion of TMP can be used in high quality wood-containing papers, i.e. LWC or SC grades, without serious printability problems.

It has been shown that the degree of fibre collapse during refining under standard TMP conditions is strongly related to fibre-wall thickness (Kure 1999). However, the wall thickness of the fibres with the thickest walls cannot easily be changed by peeling actions in the refining process under standard TMP conditions, within the CSF range of interest for news and magazine grades. Therefore it is obvious that the refining conditions have to be modified to be able to collapse these fibres properly. With a good screen room design, most of the thick-walled fibres from the main line can be collected in the reject refiner, where the degree of fibre collapse is enhanced. In earlier studies (Norgren et al. 2004) we have shown that thick-walled fibres are more easily collapsed by reject refining after preheating to temperatures well above the softening temperature of lignin, than by refining under standard conditions.

If water is applied to the surface of a paper during coating or printing, some fibres decollapse, i.e. revert towards their original shape. This is believed to be caused by stress relaxation and fibre swelling (Skowronski, Lepoutre 1985). In the current study, the effect of the preheating temperature in reject refining on the decollapse of coarse fibres is evaluated. This was studied using an
ESEM microscope, where changes in fibre dimensions in laboratory sheets were monitored during a moisturising cycle. When using the ESEM technique, as described by Norman (Norman, Höglund 2003), it is possible to quantify the degree of irreversible fibre collapse. However, this method involves time-consuming fibre and data analyses. Therefore a complementary method has been developed and is described in this paper. The new method gives information about the effect of moisture on the smoothness of laboratory sheets. The main advantage of the procedure is its simplicity. In this investigation some other effects are also quantified using different well-established methods to give a more fundamental understanding of how temperatures well above the softening temperature of lignin affect fibre and sheet surface properties.

\section{Experimental}

High temperature, HT, trials were carried out on highfreeness screen reject with a large content of long fibres, at the Ortviken TMP plant in Sundsvall, Sweden (Norgren et al. 2004; Engstrand, Sundblad 1987).

Reject refining took place after a short preheating to temperatures below and well above the softening temperature of lignin i.e. reference pulp to $135^{\circ} \mathrm{C}$ and HT pulp to $175^{\circ} \mathrm{C}$ respectively. The effects of preheating to temperatures below and above the softening temperature of lignin were compared. The effect on fibre and surface properties of spruce TMP reject was studied using several methods that are described below.

\section{Pulp and paper testing}

Pulps from high temperature and reference trials were analysed using several methods. Many methods make use of $65 \mathrm{~g} / \mathrm{m}^{2}$ laboratory sheets, made according to the Rapid-Köthen method (ISO 5269-2). In this method laboratory sheets are dried at $95^{\circ} \mathrm{C}$ using an applied pressure of $96 \mathrm{KPa}$. In the Rapid-Köthen method the effect of lignin softening is taken into consideration in the consolidation of the sheet. This is especially important for pulps with a large content of lignin, such as TMP pulp. The following methods were used:

COD: Determination of chemical oxygen demand in water-CODCR oxidation with dichromate was done according to method (SS 0281 42). Pulps were washed according to a STFI method.

Split fibre: This method is a PFI method described in (Reme et al. 2002). PFI used a SEM microscope in BEI mode, at a resolution of $0,3 \mu \mathrm{m} / \mathrm{pixel}$. Images were editing manually and about 1000 fibres were measured for each 
sample. The results presented using this method are based on mean fibre-wall thickness for fibres with intact cell walls, fibres with a split cross-section and the total wall thickness for both intact and split fibres.

Simon Stains: Simon Stains method gives information about how fibre-walls have been treated. The principle is that pigments of a certain size penetrate the fibre-wall to different extents (Simons 1950; Yu et al. 1995).

ESCA: ESCA (Electron Spectroscopy for Chemical Analysis) is a well-known method, also known as XPS (X-ray Photoelectron Spectroscopy), to measure the chemical structure of surfaces. Using this method, the surface composition and surface coverage of lignin, carbohydrates and extractives, respectively, were estimated (Carlsson 1996; Österberg 2000).

ESEM: The ESEM (Environmental Scanning Electron Microscope) has been used successfully in earlier studies to visualise the effect that changes in moisture have on the shape of single fibres and the fibre matrix of a sheet (Grön, Berghello 1996; Hornatowska et al. 1999; Omholt 2000; Omholt et al. 1994; Sheehan, Scriven 1991). Using this technique it is possible to quantify the phenomenon that causes sheet roughening, from measurements of transverse fibre instability, when fibres are moisturised. Norman (Norman, Höglund 2003) introduced a new tool and concept to detect how fibres decollapse. RapidKöthen sheets of long fibre fractions were made, and then placed into the ESEM instrument and pictures were taken of the same area before and after the moisturising cycle. Fig 1 shows how the method records the changes in the shape of the same fibres.

The MID (moisture induced fibre decollapse) describes how single fibres change during the moisturising cycle. From ESEM pictures such as the one in Fig 1, the measurements of fibre dimension are made, as described in Fig 2. Dimensions were measured with digital software, Image-Pro plus by drawing an arrow from side to the other side when for example measuring the fibre

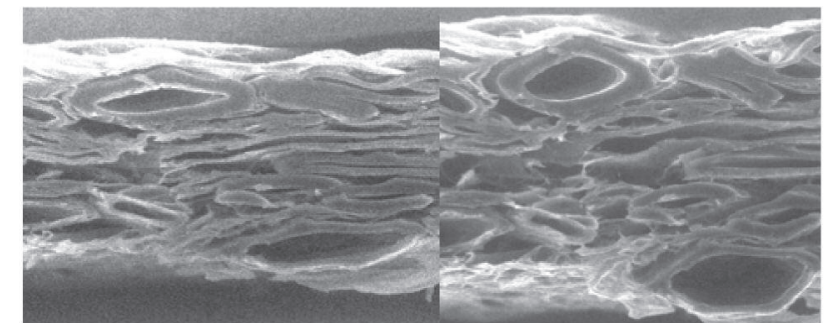

Fig 1. ESEM pictures of the same fibres before (left) and after (right) moisturising. Figure from Norman (Norman, Höglund 2004).

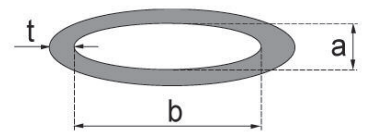

a - fibre lumen height

b - fibre lumen width

t - fibre-wall thickness

Fig 2. Fibre thickness, lumen height and lumen width were measured before and after moisturising the same fibre. height. Were on the fibre and what fibre was subjectively chosen. About 500 fibres/sample were measured. From those measurements the MID was calculated, according to $E q 1$.

$$
M I D=a_{w e t}-a_{d r y}
$$

$a_{\text {wet }}$ Lumen height after moisturising and drying of the sample

$a_{d r y}$ Lumen height before moisturising of the sample

Moisturised roughening: This is a new method that has been developed. With this technique one can detect how moisture affects the surface of a paper sheet. The method set-up is briefly described in the block diagram in Fig 3. The system requirements are a conditioning chamber that can regulate up to $98 \%$ R.H with a temperature of $23^{\circ} \mathrm{C}$. A good pair of scales is also necessary and a non-contact instrument to measure surface roughness.

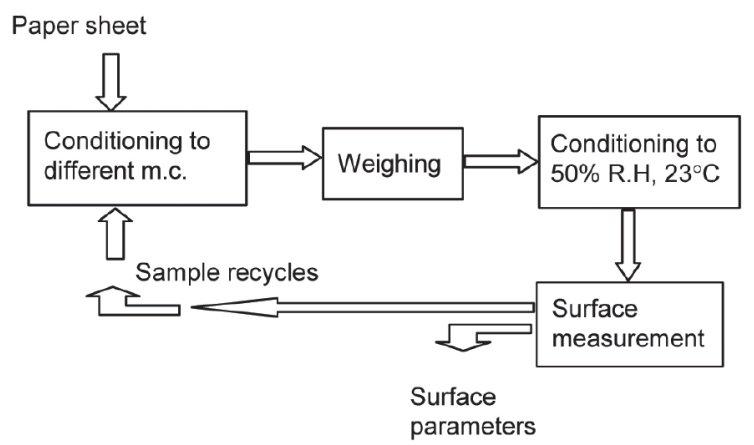

Fig 3. Block diagram of the method, moisturised roughening.

A single sheet was suspended from a pair of scales down into a climate chamber. Paper sheets were then conditioned at a certain relative humidity for $2 \mathrm{hs}$. The weight of the sheet was then measured. The paper sheet was then placed in a climate room with a temperature of $23^{\circ} \mathrm{C}$ and relative humidity of $50 \%$. In that room an optical scanning device to measure surface roughness was placed. After room conditioning for about $8 \mathrm{hs}$ the surface roughness measuring could take place. Surface measurements were made on three areas of $1 \mathrm{~cm}^{*} 1 \mathrm{~cm}$ on the paper sheet with an image size of $1000 * 1000$ points. As Fig 3 shows the moisturising process was repeated. The same paper was conditioned several times to higher and higher moisture contents. The procedure with weighing, room acclimatisation and surface measurements was also repeated.

After the last surface measurement on the sheet, the sheet was dried at $105^{\circ} \mathrm{C}$ in an oven. Then the dried sheet was weighed and the dryness at every point on which measurements had been made could be calculated. Note that the same areas of the sample are used, but changes in the moisture level create new surfaces.

The surface measurement system used, hereafter-called FRT, is manufactured by the Fries Research and Technology company. The main difference compared to other surface measurement systems is the use of a cheap 


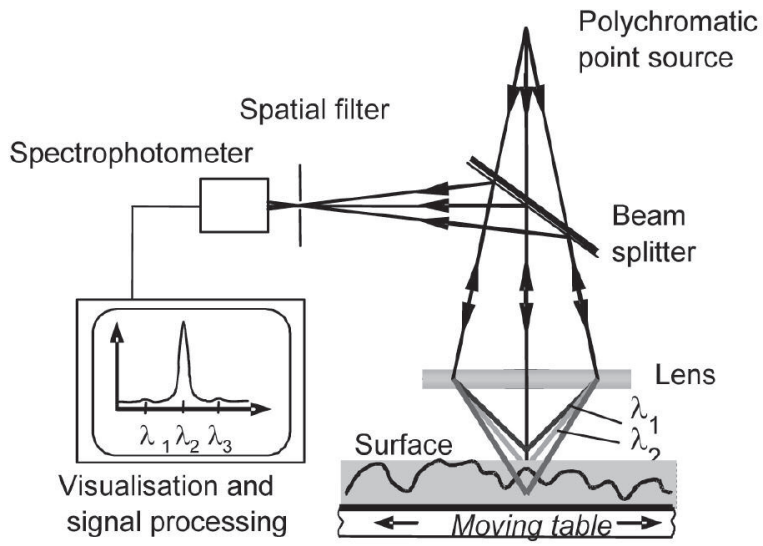

Fig 4. The principle of the surface measurement system.

polychromatic light source. The light source provides the optical system with light via fibre optics, which shine through a small optical system consisting of lenses that enhance the chromatic aberrations, which means that the different wavelengths of light will not focus on the same point, but that different wavelengths will have their focusing points in different positions. This aberration normally creates blurriness in an image, but here it is a powerful property used to determine the surface topography. The principle of the surface measurement system is illustrated in Fig 4.

\section{Result and Discussion}

The basic idea of the project was to soften TMP reject before refining at a temperature well above the softening point of lignin. Using this method the expectation was that fibres would collapse irreversibly to a higher extent than under standard reject refining conditions. With a higher degree of irreversible collapsed fibres, it would be possible to increase the proportion of TMP in high quality wood-containing printing papers. In a previous paper, IMPC 2003 (Norgren et al. 2004), the high temperature reject refining process was described in more detail and the process was shown to be energy effective. It was also shown that the content of shives and $\mathrm{BMN}+10$ fraction was reduced, a higher degree of fibre collapse was achieved and sheet roughness was reduced.

This paper focuses on other effects of high temperature reject refining. This will give a more complete picture and understanding of fibre behaviour and also of how surface properties changes during the process of reject refining. The results are presented in four parts. The investigation that is reported in the first part was carried out in order to ensure that the HT process did not lead to any significant reduction in yield and also that no significant reduction in brightness took place. Part two focuses on fibrewall properties. These have been investigated by making measurements of reference samples and HT-process samples. Part three shows how moisture changes fibres and surface structures. In part four, differences in the chemical composition of the fibre surfaces are compared in the two reject refining processes.

\section{Pulp Properties}

After STFI washing of reject refined pulp in the freeness range 120-200 $\mathrm{ml}$, COD-values from the two processes were compared. The COD averages in Table 1 show that high temperature may give a somewhat higher loss in yield compared to the reference reject refining process. However, the difference is not significant.

Table 1. COD according to method by dr Lange.

\begin{tabular}{cc}
\hline Samples & COD average $(\mathbf{k g} / \mathbf{t})$ \\
\hline HT & $20,2 \pm 3,5$ \\
Reference & $19,8 \pm 2,0$ \\
\hline
\end{tabular}

Another important property of pulp is its brightness. The use of high temperatures has the disadvantage that it gives a small reduction in brightness, but as Fig 5 shows, in furnishes for making high quality magazine paper, such as LWC, the brightness reduction is almost negligible. One reason for that is the low light scattering of reject pulps in comparison with pulps from screen accepts. In the example illustrated in Fig 5 the HT reject has about 2-units lower luminance value than the reference reject pulp. Three LWC furnishes were made from mixtures of unbleached TMP that include the two reject pulps. The figure show that it is only the furnish with $15 \%$ kraft pulp that might have a slightly higher luminance value in the mixture with reference reject pulp.

\section{Effects of fibre wall properties}

In the investigation reported earlier (Norgren et al. 2004), it was shown that the fibre fraction with the largest wall thickness was most positively affected by the HT-reject refining. In view of those results, several new measurements were made to discover how high-temperature reject refining affects different fibre properties.

In Fig 6, distribution curves of split fibres and intact fibres are shown for high-temperature reject pulp and the reference pulp. A split fibre is a fibre with a broken fibre-wall. There were no significant differences as a result of the two processes. $29 \%$ split fibres were detected in HT pulp and $26 \%$ in the reference pulp respectively. It can be noticed that it is mainly the fibres with thin walls that are split in reject refining. Almost all fibres

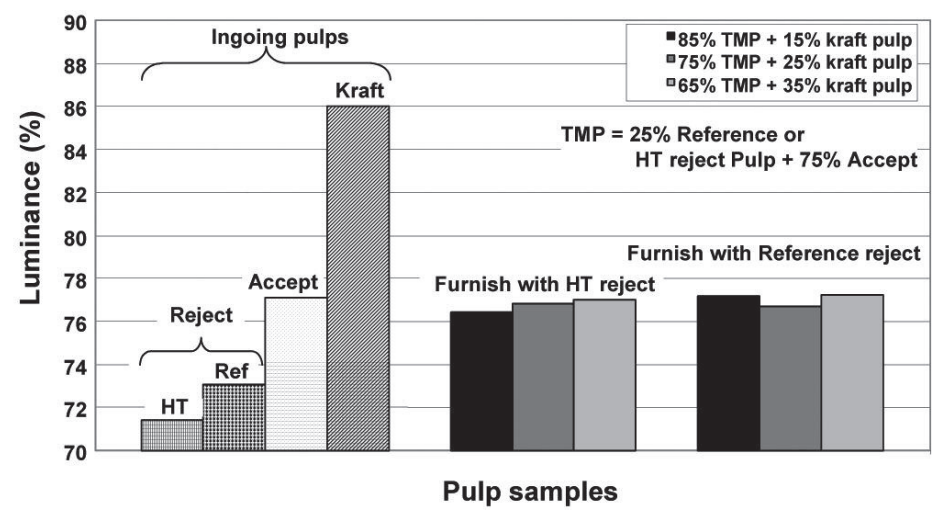

Fig 5. Luminance of pure samples and LWC furnishes. 


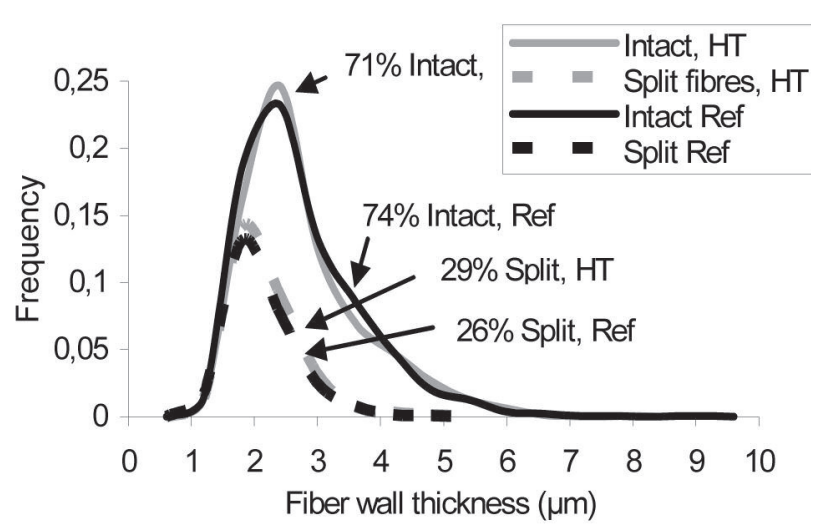

Fig 6. Amount of Split fibres in HT refined pulp and in reference pulp.

with a wall thickness $>3 \mu \mathrm{m}$ have intact fibre-walls in this respect. Another fibre-wall analysis, the Simons stain, has been used to complement and verify that high temperature does not affect fibre-wall structure negatively. In the Simons stain method, fibres are examined in a light-microscope and then divided into different groups according to how the fibre-wall has been affected. The fibres are divided into the following groups:

Blue: $\quad$ Unaffected fibres.

Blue-Yellow: Partly affected fibre-walls

Green: Homogeneously affected fibre-walls

Yellow: Totally affected fibre-walls

*Blue-Yellow: Fibres in which the tail end has been

split . apart like a brush

From Table 2 it can be seen that there was no major difference in how the two refining processes affected the fibre-walls, in spite of the fact that the disc gap is narrower in HT refining.

Table 2. Simons stain analysis of 2000 fibres of each sample, from the reference and the high-temperature reject refining processes.

\begin{tabular}{lccccc}
\hline Sample & $\begin{array}{c}\text { Blue } \\
(\%)\end{array}$ & $\begin{array}{c}\text { *Blue / } \\
\text { Yellow (\%) }\end{array}$ & $\begin{array}{c}\text { Blue / } \\
\text { Yellow (\%) }\end{array}$ & $\begin{array}{c}\text { Green } \\
(\%)\end{array}$ & $\begin{array}{c}\text { Yellow } \\
(\%)\end{array}$ \\
\hline High temperature & 46 & 6,5 & 44 & 2 & 1,5 \\
Reference & 43 & 6 & 47 & 2,5 & 1,5 \\
\hline
\end{tabular}

\section{Effects of fibre and sheet roughening}

The measurements of the fibre-wall structure showed that it was not significantly changed by the high preheating temperature in the reject refining system. It will be shown below that the main effect is a change in the shape of the fibres and the degree of irreversible fibre collapse. The degree of irreversible fibre collapse was measured using the ESEM method and described as a MID value.

The results are shown in Fig 7. In each series in the figure approximately 500 fibres were measured according to the method illustrated in Figs 1 and 2. The highest MID-values were found in fibres with the highest wall thickness i.e. it is most difficult to get the most thick-walled fibres to collapse irreversibly. The MID-values also show that HT reject fibres are more irreversibly collapsed than reference fibres. The irreversible collapsing effect caused by high temperatures decreases with fibre-wall thickness, as illustrated in Fig 7

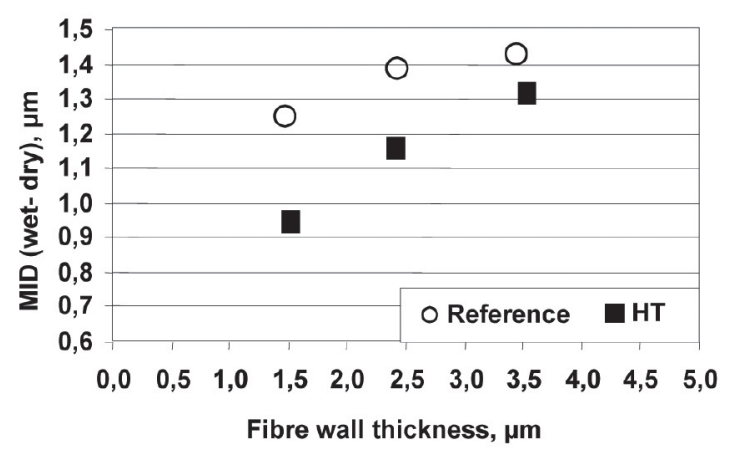

Fig 7. MID (Moisture induced fibre decollapse) vs. wall thickness. Pulps are in the freeness range 140-180 ml CSF.

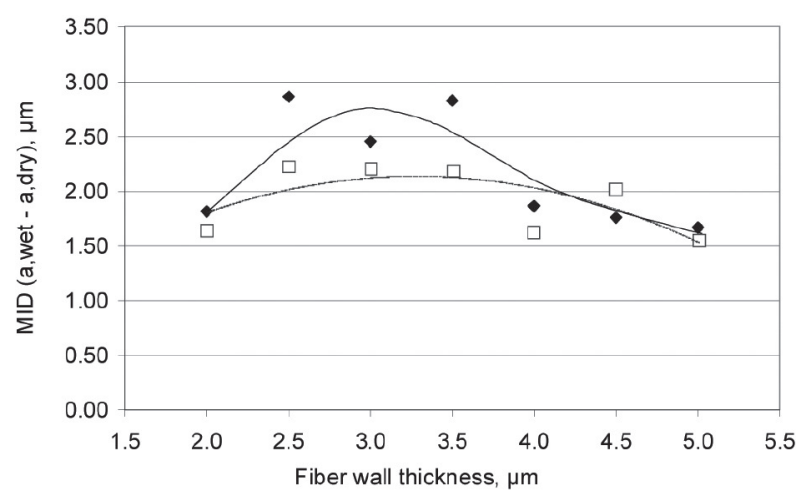

Fig 8. MID (Moisture-induced fibre decollapse) vs. fibre-wall thickness from main line refining (filled diamond) and disc filter pulp (open square). Figure by Norman (Norman, Höglund 2003).

Fig 8 above is taken from Norman (Norman, Höglund 2003). It shows the MID behaviour of Norwegian spruce TMP in different process stages. The maximum MIDvalue on fibres appear after main line refining (Fig 8). The maximum was located in the region of early wood fibres with relatively thick fibre-walls and transition wood fibres. MID was clearly lower in the disc filter pulp i.e. screened and reject refined pulp.

A comparison between values from Fig 8 and MID values from the two reject refining processes, Fig 7, that are investigated in the current study, shows that the MID values are effectively reduced as a result of reject refining. As expected, it is most difficult to irreversibly collapse the fibres with the thickest fibre-walls, although even those are most effectively treated after softening at a high temperature.

With the new method "Moisturised roughening" it is easy to monitor surface roughness, when moisture is applied to a paper sheet. Fig 9 illustrates how moisture affects the roughness value, $R a$, of paper sheets. How moisture affects paper sheets from the two refining processes differently is also illustrated. The paper sheet made from HT-pulp has somewhat lower roughness. This shows that the new method also indicates how the degree of decollapse is related to moistening. It is worth remembering that, in the ESEM method, fibres are wetted to a saturated state, whereas in the "Moisturised roughening" method fibres in paper sheets are exposed to moisture in a climate chamber at a certain $\mathrm{RH}$ value. 


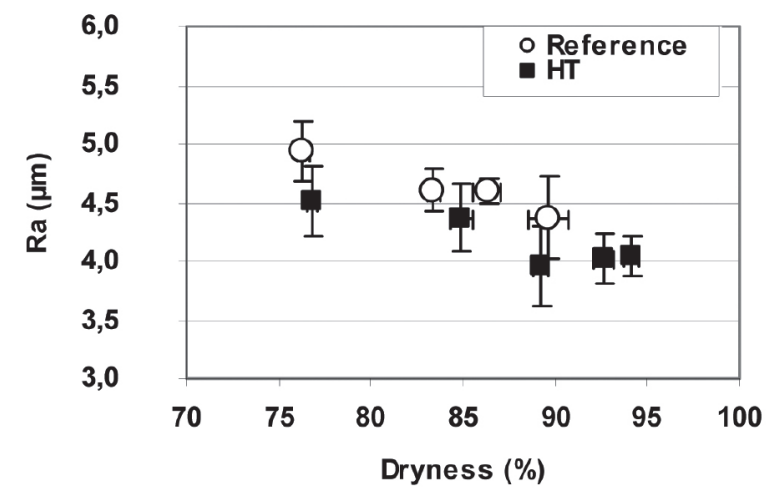

Fig 9. Ra values of Raphid-Köthen sheets that have been conditioned to higher and higher moisture in several stages.

\section{Effects of surface properties}

The surface compositions of some selected pulp samples were analysed using electron spectroscopy for chemical analysis (ESCA or XPS). This method measures the atomic composition of the outmost surface layers, into a maximum depth of analysis of $100 \AA$.

Table 3 shows the per centage of carbon and oxygen atoms in the surface of pulp sheets. Naturally, $\mathrm{C}$ and $\mathrm{O}$ dominate the spectra, some traces of $\mathrm{N}$ and $\mathrm{Ca}$ could also be seen. The difference between the pulp samples was judged to be small, even in the detailed analyses of the spectra. The table shows that the unextracted samples were quite similar. Extraction gave a general increase in the $\mathrm{O} / \mathrm{C}$ ratio, as expected, since extractives contain much less oxygen in relation to carbon than lignin or cellulose (Carlsson 1996).

At both freeness levels, there was a slight trend towards an increased $\mathrm{O} / \mathrm{C}$ ratio in the extracted high temperature pulp compared to the extracted reference pulp. This indicates a somewhat higher amount of oxygen in the surface of the high temperature pulps. This is probably not due to increased proportions of hemicelluloses or cellulose, since the surface coverage of lignin calculated, based on the ESCA-spectra, did not show any significant differences between the samples. It is more likely that the lignin is modified during HT reject

Table 3. Calculated surface coverage values of reference and HT samples respectively.

\begin{tabular}{lccc}
\hline Sample & \multicolumn{2}{c}{ Atom $\%$} & Atomic ratio \\
\hline & C & 0 & $0 / C$ \\
Reference, 141 ml CSF & 63.8 & 35.9 & 0.56 \\
Unextracted & 60.5 & 38.5 & 0.64 \\
Extracted & & & \\
& & & \\
HT, 151 ml CSF & 63.6 & 36.0 & 0.57 \\
Unextracted & 59.9 & 39.8 & 0.66 \\
Extracted & & & \\
& & & \\
Reference, 190 ml CSF & 64.1 & 35.2 & 0.55 \\
Unextracted & 60.7 & 38.4 & 0.63 \\
Extracted & & & \\
HT, 185ml CSF & & & 0.55 \\
Unextracted & 63.9 & 35.3 & 0.67 \\
Extracted & 59.6 & 39.7 & \\
\hline
\end{tabular}

refining. The effect was noted but has to be further investigated before any conclusions can be drawn from the data.

\section{Conclusions}

High temperature reject refining is a suitable method for reducing the problem of surface roughening in TMP based sheets. Earlier studies have shown that the process reduces the energy consumption and also that a higher degree of fibre collapse is achieved. This complementary study shows that the technique of high temperature reject refining compared to refining under standard conditions, concludes that:

- A higher degree of irreversible fibre collapse.

- No significant changes in the surface composition of the fibres.

- No significant changes in fibre-wall structure according to measurements of split fibres or Simons stain analysis.

- Very small changes in yield.

- Marginal effects on brightness in the final paper.

\section{Future activities}

This study will be followed by further development of the moisturised roughening method. It will be used to investigate how different fractions of TMP reject pulp influence roughness.

\section{Acknowledgements}

Many people have contributed to the results of this study. We would like to take this opportunity to thank:

*SCA Ortviken, for facilitating full-scale trials.

*SCA Research, especially Roland Bäck and Christina Westerlind, for their invaluable co-operation.

*Mats Rundlöf, for valuable comments on surface measurements.

*Mimi Finnstedt, for the linguistic revision of the manuscript.

*The Swedish Energy Agency, the Foundation for Knowledge and Competence and the European Union, Objective 1, Region of South Forest Counties for the financial support.

\section{Literature}

Carlsson, G. (1996): Surface composition of wood pulp fibres: "Relevance to wettability, sorption and adhesion", Department of Pulp and Paper Chemistry and Technology, Royal Institute of Technology and Institute for Surface Chemistry, Stockholm, Sweden.

Engstrand, B. and Sundblad, P. (1987): Ortviken - The Uncommon Paper Mill, In: International Mechanical Pulping Conference, Vancouver, pp. 91-97.

Forseth, T. and Helle, T. (1996): Effect of moistening on cross sectional details of calendered mechanical paper, 82nd Annual Meeting Technical Section, pp. A273A280.

Forseth, T. and Helle, T. (1998): Moisture-induced roughening during "water coating" of precalendered wood-containing paper, J. Pulp Paper Sci. 24(10), pp. 301-307.

Forseth, T., Wiik, K. and Helle, T. (1997): Surface roughening mechanisms for printing paper containing mechanical pulp, Nord. Pulp Paper Res. J. 12(1), pp. 67-71.

Grön, J. and Berghello, L. (1996): Cracking and roughening of coated paper surfaces, Paperi ja Puu, 78(3), pp. 121-127. 
Hornatowska, J., Östlund, S. and Fellers, C. (1999): ESEM investigations of damage at defects in newsprint, Microscopy as a tool in pulp and paper research and development, STFI, Stockholm, Sweden, pp. 59-71.

Kure, K-A. (1999): On the relationship between process input variables and fibre characteristics in thermomechanical pulping, Institutt for kjemisk prosessteknologi, NTNU, Trondheim, Norway.

Norgren, S., Höglund, H. and Bäck, R. (2004): Irreversible long fibre collapse at high temperature TMP reject refining - initial studies, Pulp Paper Can. 105(7), pp. 170-174.

Norman, F. and Höglund, H. (2003): Moisture-induced surface roughness in TMP-based paper - the influence of fiber cross-section dimensions, In: International Mechanical Pulping Conference, Quebec, Canada, pp. 57-83.

Omholt, I. (2000): Characterization of TMP fibers by scanning electron microscopy, In: VTT Third workshop on measuring of wood properties, grades and qualities in the conversion chains, Espoo, Finland.

Omholt, I., Forsberg, P. and Lepoutre, P. (1994): ESEM examination of the roughening of paper in high moisture environment, In: International printing and graphic arts conference.

Reme, P.A. (2000): Some Effects of Wood Characteristics and the Pulping Process on Mechanical Pulp Fibres, Institutt for kjemisk prosessteknologi, NTNU, Trondheim, Norway.
Reme, P.A., Johnsen, P.0. and Helle, T. (2002): Assessment of Fibre Transverse Dimensions using SEM and Image Analysis, J. Pulp Paper Sci. 28(4), pp. 122128.

Sheehan, J.G. and Scriven, L.E. (1991): Assessment of environmental scanning electron microscopy for coating research, In: Coating conference, TAPPI Proceedings.

Simons, F.L. (1950): A stain for use in the microscopy of beaten fibers, Tappi, 33(7), pp.312-314.

Skowronski, J., Lepoutre, P. (1985): Water paper interaction during paper coating changes in paper structure, Tappi J. 68(11), pp. 98-102.

Yu, X., Minor, J.L., Atalla, R.H. (1995): Mechanism of action of Simon's stain, Tappi J. 78(6), pp. 175-180.

Österberg, M. (2000): On the interactions in cellulose systems: Surface forces and adsorption, Department of Chemistry, Surface Chemistry, Royal Institute of Technology, Stockholm, Sweden, Department of Forest Products Technology, Helsinki University of Technology, Helsinki, Finland and Institute for Surface Chemistry, Sweden.

Manuscript received July 7, 2008 Accepted November 13, 2008 